\title{
Des hommes aux champs
}

Alain Ferdière

\section{OpenEdition}

Journals

Édition électronique

URL : http://journals.openedition.org/rao/2235

DOI : 10.4000/rao.2235

ISBN : 978-2-7535-3432-2

ISSN : 1775-3732

Éditeur

Presses universitaires de Rennes

Édition imprimée

Date de publication : 25 décembre 2013

Pagination : 318-320

ISBN : 978-2-7535-3430-8

ISSN : 0767-709X

\section{Référence électronique}

Alain Ferdière, "Des hommes aux champs », Revue archéologique de l'Ouest [En ligne], 30 | 2013, mis en ligne le 25 décembre 2013, consulté le 03 décembre 2020. URL : http://journals.openedition.org/ rao/2235; DOI : https://doi.org/10.4000/rao.2235

Ce document a été généré automatiquement le 3 décembre 2020.

Tous droits réservés 


\title{
Des hommes aux champs
}

\author{
Alain Ferdière
}

\section{RÉFÉRENCE}

Carpentier V. et Marcigny C. (dir.), 2012 - Des hommes aux champs. Pour une archéologie des espaces ruraux du Néolithique au Moyen Âge, (Actes de la table ronde des 8-9 oct. 2008, Caen), Rennes, PUR, 459 p. + cahier central de XLVIII Pl. couleur h. t. (ISBN

978-2-7535-2101-8, $35 €)$

1 Cet imposant volume constitue donc la publication de la première table ronde intitulée «Des hommes aux champs» (2008). Jouant d'emblée sur le double sens du titre, la table ronde comme ses actes se veulent en outre résolument diachroniques, sur le temps long - comme l'indique le sous-titre -, ainsi que largement interdisciplinaires, même si l'entrée est prioritairement celle de l'archéologie.

On sait qu'une seconde table ronde s'est tenue sous le même titre à Caen en septembre 2011 : j'en avais fait un rapide compte-rendu dans le Bulletin AGER n 21 (2012, p. 29-31).

Comme spécialiste du monde rural gallo-romain, je n'ai pas la prétention de rendre compte ici du détail des contributions pour les périodes qui me sont plus étrangères, si ce ne sont, à la rigueur, les marges de la période romaine, à La Tène finale et au très Haut Moyen Âge.

4 Passée une suggestive Introduction signée des co-directeurs de la publication (coorganisateurs de la Table Ronde), Vincent Carpentier et Cyril Marcigny, le Sommaire se divise ainsi en deux grandes parties à peu près égales (environ 200 pages chacune) : la première est constituée de contributions classées dans l'ordre chronologique, pour le Néolithique (3 contributions), l'Âge du Bronze (1), le Second Âge du Fer (2), la période romaine (3), le Haut Moyen Âge (3), et le reste de la période médiévale (3). La seconde comprend des travaux sur la longue durée, à des échelles d'espaces diverses.

Une fois traité donc du Néolithique, qui marque évidemment la genèse des phénomènes examinés ici (l'exploitation des espaces ruraux), il est notable que, comme il fallait s'y attendre, la Protohistoire ancienne (Âge du Bronze, Hallstatt, La Tène Ancienne...) soit 
sous-représentée: ces questions ne sont clairement pas dans les préoccupations principales actuelles des spécialistes de ces périodes, tournées plus volontiers vers les questions d'habitat, de métallurgie, de typologie céramique, et culturelles...

6 La notion de territoire, avec une vision spatiale des phénomènes, est par ailleurs au cœur de bon nombre de ces études. La prise en compte des données de l'archéologie préventive est notable dans plusieurs de ses contributions. Et l'on note aussi bon nombre de ces travaux qui intègrent utilement les données paléoenvironnementales et bio-archéologiques, dans une vision intégrée des systèmes agraires.

Ce sommaire copieux (22 contributions auxquelles s'ajoutent Introduction et Conclusion) mérite d'être détaillé et rapidement examiné contribution par contribution :

Des études chronologiques... :

-J. Dubouloz, «Interdépendance et cohésion des différents niveaux de territorialité au Néolithique Rubané en Bassin Parisien", p. 23-34, avec tentatives de modélisation, à différentes échelles, au Néolithique.

-Y. Lanchon ( $\dagger)$, «Le Néolithique Ancien dans la basse vallée de la Marne. Premières réflexions sur les sites, leur statut et leur organisation territoriale ", p. 35-54, ici aussi avec essais de modélisation des territoires pour le Néolithique, s'interrogeant notamment sur les ressources.

-E. Ghesquière, «Essai d'analyse d'un espace rural au Néolithique Moyen II. L'exemple de la plaine nord d'Argentan ", p. 55-70, pour un espace de superficie comparable, concernant la vallée de l'Orne, avec en particulier l'exploitation du silex.

- C. Marcigny, « Les paysages ruraux de l'Âge du Bronze (2300-800 avant notre ère). Structures agraires et organisations sociales dans l'Ouest de la France », p. 71-80, très synthétique mais suggestif, notamment quant à cette question de structures agraires et paysages ruraux, s'appuyant sur les opérations préventives.

- G. Blancquaert et M. Derreumaux, "L'occupation spatiale au second Âge du Fer dans le Nord-Pas-de-Calais », p. 81-98, première et intéressante contribution pour la période laténienne (avec une redondance dans le titre car une occupation ne peut être que spatiale, et malheureusement encore une fois sur un territoire administratif actuel peu pertinent pour la période); les données bio-archéologiques sont intégrées, pour l'étude de l'espace rural et des ressources.

- G. San Juan, «L'évolution des formes de l'habitat protohistorique au nord-ouest de Caen. L'exemple du plateau de Thaon-Basly après dix années de recherche programmée ", p. 99-109, très synthétique aussi, sur une unique commune de la vallée de la Mue, étudiée intensivement en particulier grâce à l'archéologie programmée.

-F. Favory, «Les parcellaires antiques de Gaule médiane et septentrionale », p. 111-130, utile bilan pour la période romaine ( $1^{\text {re }}$ contribution ici pour cette période) et cette parfois épineuse question des parcellaires, ici certes fondé sur la photo-interprétation (notamment en milieu forestier) mais aussi sur des données de fouilles extensives et particulièrement préventives; on note des différences micro-régionales; la prégnance des normes romaines subsiste dans les recherches.

-P. Ouzoulias, «Les campagnes gallo-romaines: quelle spatialité ? Retour sur l'expérience d'Archaeomedes ", p. 131-155, analyse perspicace, de caractère historiographique, avec le recul nécessaire, pour les principales publications issues de ce programme (portant essentiellement sur la Narbonnaise) : relations société-milieu, sociétés rurales et systèmes productifs... 
- M. Georges-Leroy, J. Bock, É. Dambrine, J.-L. Dupouey, A. Gebhardt, J.-D. Laffite, « Les vestiges gallo-romains conservés dans le massif forestier de Haye (Meurthe-et-Moselle). Leur apport à l'étude de l'espace agraire ", p. 157-180, un bon cas d'étude, récente, sur la conservation de ces traces en milieu forestier, surtout de parcellaires de pierre, pour la Côte de Moselle, avec application du LIDAR.

- V. Carpentier, «Premières réflexions sur la place des enclos mérovingiens de la plaine de Caen dans l'évolution des espaces ruraux de la Basse-Normandie sur le temps long", p.181-193, assez synthétique, qui se place dans une perspective de résilience et de diachronie, et prenant encore en compte les données de l'archéologie préventive: on reconnaît des différences micro-régionales ; les relations avec le climat sont évoquées.

-É. Peytremann et J. Wiethold, « L'apport de la carpologie à l'étude du site du Premier Moyen Âge (VI'-XII ${ }^{\mathrm{e}}$ siècle) de Semersheim (Bas-Rhin)», p. 195-212, l'une des rares études de cas d'un site unique site de la vallée de l'Ill, quant à ses productions et son environnement à travers les données paléoenvironnementales et bio-archéologiques, notamment de la carpologie.

-F. Guérin, «Note sur trois installations rurales du Bas Moyen Âge fouillées à SaintBarthélemy-d'Anjou (Maine-et-Loire)», p. 213-225, ici courte étude de cas de trois sites perçus à partir d'un diagnostic de 92 ha, avec occupation surtout au Haut Moyen Âge : étude de terroir.

- C. Hanusse, «Les habitats désertés au Moyen Âge. Quelques réflexions à propos d'un paradigme", p.227-235, bref retour sur un vieux questionnement de l'archéologie médiévale française, article de caractère surtout méthodologique concernant la question du «village déserté » : apports récents de l'archéologie préventive, fournissant de fait peu de sites $\mathrm{du} 2^{\mathrm{e}}$ Moyen Âge; s'agit-il d'un problème d'échelle d'observation? Une vision dynamique de l'occupation du sol est nécessaire, pour comprendre la maîtrise de l'espace par les sociétés médiévales.

... Aux études sur la longue durée :

-D. Flotté, L. Ménager et L. Vipard, « Contribution du diagnostic archéologique à l'archéologie des espaces ruraux », p. 239-261, qui se pose la question, fondamentale, de l'exploitation des données de diagnostic (sous-exploité, ce qui n'est pas vraiment une « surprise »), bien que malgré le titre - sur un espace réduit, en Basse-Normandie (trois espaces diagnostiqués).

-É. Cavanna, «L'espace, un estimateur archéologique de distinction sociale ? L'exemple des élites médiévales et modernes "aux champs" ", p. 263-274, au titre conceptuellement un peu obscur, est de caractère "épistémologique » et méthodologique, un peu sous forme de déclarations d'intentions (thèse en cours).

- M. Watteaux, «Archéogéographie morphologique de la Plaine sud-vendéenne », p. 275-287, autour de la "nouvelle » discipline de l'« archéogéographie », qui ne peut être en effet que « morphologique » : la période laténienne est privilégiée; le terme à la mode mais parfois pertinent de « résilience » est utilisé...

-L. Lespez, C.Germain-Vallée, C. Riquier, «Archéologie du paysage en plaine de Caen. Nouveau programme et premiers résultats", p. 289-303, utilisant le concept plus ancien mais non obsolète d'«archéologie du paysage », présentant surtout les intentions d'un programme dont les résultats restent à conforter: déclaration d'intentions et premiers résultats d'un PCR sur la Plaine de Caen, avec SGBD et mise en œuvre des opérations préventives.

-D. Schwartz, D. Ertlen, J. Battmann, M. Caspard, A. Geghaert, S. Goepp, F. Basoge, L. Koupaliantz, B. Metz, «Études actuelles sur un type de paysage encore très peu connu en Alsace: les paysages d'enclos médiévaux. Extension, typologie, éléments de datation », 
p. 305-326, au titre assez maladroit, pour des structures elles aussi bien conservées en sousbois : paysages enclos, avec des problèmes de datation et des différences micro-régionales.

- M.-P. Ruas, V. Zech-Matterne, «Les avoines dans les productions agro-pastorales du NordOuest de la France. Données carpologiques et indications textuelles », p. 327-365, seconde contribution mettant en œuvre la carpologie, ici sur le temps long, avec études de cas et examen des textes, spécifiquement pour l'avoine, à partir de l'Âge du Fer et surtout au Moyen Âge : relations avec l'économie rurale et les questions de fourrage.

-F. Malrain et D. Maréchal, «Espaces ruraux dans la moyenne vallée de l'Oise. Limites et perspectives ", p. 367-390, pour un secteur très concerné par l'archéologie préventive et sur le temps long, du Néolithique à l'époque romaine (et surtout La Tène-gallo-romain, avec absence ici du Moyen Âge...), dont discussion sur l'étendue des exploitations.

-J.-M. Séguier, « Fermes, terroirs et territoires du Deuxième Âge du Fer à l'époque romaine dans le secteur Seine-Yonne (Seine-et-Marne)», p. 391-408, dans le secteur de la Bassée, article aussi très concerné par l'archéologie préventive (3000 ha perçus), mais pour une période plus courte examinée; surtout pour La Tène et la période romaine (petites fermes principalement à cette dernière période, avec des « sites intercalaires »); absence notable de parcellaires.

- B. Van den Bossche, « 2000 ans de construction des paysages agraires entre Caen et Bayeux. Aspects méthodologiques et perspectives de recherche », p. 409-433, secteur aussi de fouilles préventives, ici examinée depuis la période romaine dans son évolution, du point de vue des systèmes agraires : réseau de fermes ; traitement sous SIG et dans la diachronie; déclaration d'intentions encore et premiers résultats, avec les parcellaires et les façons culturales, surtout pour la Protohistoire.

10 S'y ajoute la Conclusion de J. Burnouf et J. Vanmoerkerke (p. 435-440), consensuelle et au propos assez général et classique. Et enfin d'utiles résumés permettent notamment aux lecteurs spécialistes d'une période donnée de prendre rapidement connaissance des avancées pour d'autres époques.

11 Ces Tables Rondes «Des hommes aux champs » n'apparaissent donc pas redondantes ou concurrentielles par rapport notamment aux Colloques AGER. Elles sont au contraire très complémentaires et salutaires, ne serait-ce que par leur caractère largement plus diachronique. Et l'accent est clairement porté - on l'a souligné - d'une part à la dimension spatiale, de l'autre aux aspects paléoenvironnementaux ; ceci sans négliger les approches plus classiques de l'ordre de l'histoire économique et sociale, ici quant aux systèmes agraires et aux productions agropastorales, avec leurs protagonistes, les « hommes aux champs »...

12 Après la publication de ce riche et suggestif volume, on attend donc avec impatience les Actes de la seconde Table Ronde de 2011, au programme copieux et diversifié aussi, dont la publication est annoncée pour 2014. 


\section{AUTEURS}

\section{ALAIN FERDIÈRE}

Professeur émérite d'archéologie, Laboratoire Archéologie et Territoires, UMR CITERES, Tours 